\title{
Analysis of Brain Tumor Disease Detection Using Convolutional Neural Network
}

\author{
Yogesh Deshmukh ${ }^{\mathrm{a}, 1}$,Samiksha Dahe a , Tanmayeeta Belote ${ }^{\mathrm{a}}$, Aishwarya Gawali a \\ Sunnykumar Choudhary ${ }^{\text {a }}$ \\ a Dept. of I.T. Sanjivani College of Engineering, Maharashtra, India
}

\begin{abstract}
Brain Tumor detection using Convolutional Neural Network (CNN) is used to discover and classify the types of Tumor. Over a amount of years, many researchers are researched and planned ways throughout this area. We've proposed a technique that's capable of detecting and classifying different types of tumor. For detecting and classifying tumor we have used MRI because MRI images gives the complete structure of the human brain, without any operation it scans the human brain and this helps in processing of image for the detection of the Tumor. The prediction of tumor by human from the MRI images leads to misclassification. This motivates us to construct the algorithm for detection of the brain tumor. Machine learning helps and plays a vital role in detecting tumor. In this paper, we tend to use one among the machine learning algorithm i.e. Convolutional neural network (CNN), as CNNs are powerful in image processing and with the help of CNN and MRI images we designed a framework for detection of the brain tumor and classifying its Different types.
\end{abstract}

Keywords. Brain Tumor, MRI, Machine Learning, Convolutional Neural Network $(\mathrm{CNN})$, Classification.

\section{Introduction}

As there is huge improvement in the medical field, medical images of various parts of human body plays a vital role in diagnostic and treatment and also in medical research. The medical imaging consists of various imaging technologies like X-ray, Fluoroscope and Magnetic resonance imaging (MRI). In all those images a tumor disease is frequent and complex. And hence Brain tumor disease detection is one of the important topics in the medical field. The detection of brain tumor is basically depending on medical imaging data analysis. The Accurate and Precise analysis of tumor is a important step in determination of patient's state. In these various factors are important as far as the correct diagnosis of tumor is concern i.e., doctor's knowledge, experience and visual weariness. And this various factor affects the accurate analysis of tumor image analysis. Hence detection of brain tumor images is very important. Magnetic Resonance Imaging (MRI)[5] provides the useful information of size, shape and position of tumor. The images obtained using MRI is accurate and exact. The efficiency of diagnosis is greatly improved with the help of MRI [5]. The MRI act as a guide for lesion localization and

\footnotetext{
${ }^{1}$ Yogesh Deshmukh, Assistant Professor, Dept. of I.T. Sanjivani College of Engineering Email:yogeshdeshmukhit@sanjivani.org.in.
} 
surgical treatment and it avoids the operations of thoracotomy. MRI of Brain uses three-dimension multi-band imaging technology [5]. In multi model MRI images we can obtain the different structures of the same tumor using the unused development sequence. Different modes can display different brain tumor features. Basically, brain tumor has classified into two different types that is Cancerous and non-cancerous. Noncancerous brain tumor is called as benign these types of tumors stays in only one place they do not spread in the body or in brain which is not that much dangerous to our body. cancerous brain tumor is called as malignant these types of tumor does not stays in only one place they spread in our body or in brain very rapidly which dangerous to our body. That's why early prediction of brain tumor is very important so that the patient can take the appropriate treatment according to its brain tumor type (i.e., cancerous and noncancerous) [4].

Magnetic Resonance Imaging (MRI) can provide information about the shape of tumor, its size, and position of the tissues and organs with no high ionizing radiation [3]. MRI of the brain is useful in identifying problems such as blurry vision or seizures, dizziness persistent headaches, weakness, and it helps in detecting certain chronic diseases of the nervous system that is multiple sclerosis. In some of the cases, we cannot able to see the clear images of parts of brain with the help of an X-ray, CAT scan, or ultrasound. that's is the reason we use MRI images here because MRI scan can provide us the precise and clear images of the different parts of the brain and makes it valuable for diagnosing. In addition, MRI imaging also can acquire totally different structures of identical tissue mistreatment the unused development sequence. That is, multimodal MRI image [3]. Different types of modes will show different tumor options.

\section{Related Work}

In the basic research, the image pre-processing is done on basis of height, width\& number of channels like RGB i.e., RED, GREEN and BLUE that means the colors, It also work with the spatial attributes. Ming Li, Lishan Kuang, Shuhua Xu \& Zhangvo Sha [1] has provide a mechanism to identify \& classify the tumor, in that they use MR image that provides information like shape, size \& position of human tissues. By using the MR image with provided information the prediction \& classification carried out. Multi-model MR image-based brain tumor detection has used by many researchers. Brain tumor detection technique is used for dividing the tumor tissues into different types such as tumor tissues, edema tissues, necrotic tissues and normal tissues. Problem of brain tumor detection has a challenge of tumor to itself, because of that it received widespread attention for tumor detection in the last 20 years. By human intervention, the image detection of brain tumor is classified into 3 categories: Manual detection, Semi-Manual section and fully-automatic Detection. The manual detection is completely manual based it is capable of manually depicting the contour of tumor. The semi-manual detection is based on the manual initialization. And the fully automatic detection is done without human interference.

\section{Problem Description and System Architecture}

Now a day's various images in medical field plays important role in diagnosis of various disease and research as well. Therefore, the research on the medical diagnosis 
data is now become very important as a brain tumor with periodic occurrences and with their complexity, now a day's brain tumor detection has become an important topic in the research of medical field. The detection of brain tumor is depending on the various medical images available on tumor. The patient's condition is determined by doing the analysis of brain tumor images accurately. So, the data available on brain tumor in the form of medical images are processed with the help of various Machine learning algorithms like Convolutional Neural Network (CNN). The MRI image provides the tumor information about size, shape and position of tumor. Those images will be provided as a input to the various machine learning algorithms. Machine learning plays vital role in prediction of brain tumor. In the Preprocessing phase the MRI images will be filter and smooth images will be given as input to the machine learning algorithm.

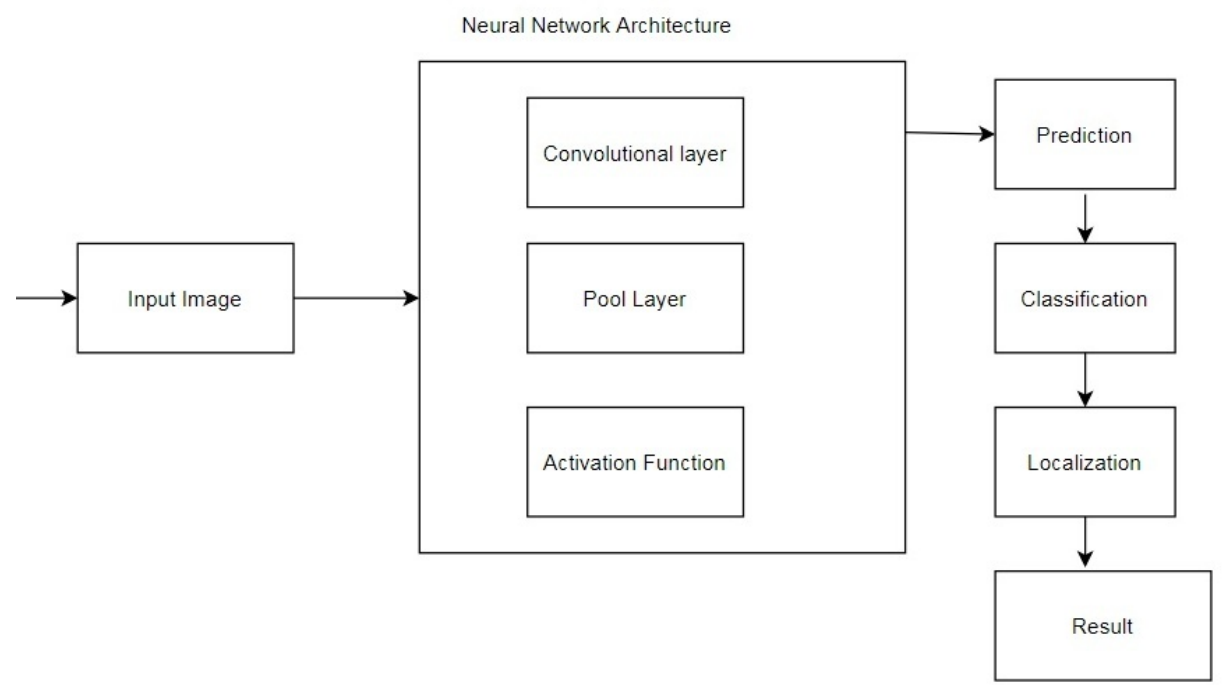

Figure 1. Architecture of the proposed system

Multi-model MR image-based brain tumor detection has used by many researchers. Brain tumor detection technique is used for dividing tumor tissues into different types such as tumor tissues, edema tissues, necrotic tissues and normal tissues. Brain tumor detection has a challenge in tumor detection to itself because of that it received widespread attention for tumor detection in last 20 years. By human intervention, the image detection of brain tumor is classified into 3 categories: Manual detection, semimanual and fully automatic detection. The manual detection is completely manual based, it is capable of manually depicting the contour of tumor, the semi-manual detection is based on the manual. Brain tumor MR images uses 3D multiband imaging technology and chest $\mathrm{x}$-ray and etc. By comparing the $2 \mathrm{D}$ images with $3 \mathrm{D}$ images, 3D multiband MRI provide the coordinate position of lesion area that helps doctors to locate the lesion area accurately.

\subsection{Input Layer:}

In this model it takes input as an 3D MR image. This MR image is the key point on basis of that MRI it will predict the presence of tumor or not. After the completion of 
pre-processing of MR images, it will be provided to the Convolutional Neural Network $(\mathrm{CNN})$.

\subsection{Convolutional Neural Network:}

$\mathrm{CNN}$ is a machine learning algorithm used very commonly in the signal processing. The name of the Convolutional Neural Network is also derived for image signals from special convolutional operations. The main purpose of convolution operation in the $\mathrm{CNN}$ is to extract features from images. Basically, CNN consisting the multiple convolutional layers, Pooled layers and fully-connected layers. Usually in the front part of the network the convolutional layer and pooled layer appearing alternately, while the latter part consisting the Fully-connected layers.

\subsection{Activation Layer:}

Activation function is a branch that is concatenated after the $1^{\text {st }}$ and $2^{\text {nd }}$ layer of CNN. The standard activation functions are namely sigmoid, ReLu and softmax.

\subsection{Pooling Layer:}

Poling is used to reduce the number of computations and it is an aggregate statistical operation of images. The main use of pooling is to remove the duplicate data or information and to reduce the dimension of feature map. After the image passes the feature map is obtained through the convolutional layer. The main purpose of pooling operation is to reduce the dimension of the feature map and to remove some redundant information thereby it is reducing the number of calculations and also avoid the overfitting. The $\mathrm{CNN}$ model predicts the tumor after the image processing. After tumor detection it simply classifies the detected tumor of appropriate type. By observation or prediction, it will classify the tumor among the four types of tumor. Namely these types are Benign, Malignant, Gliomas and Meningioma. It will help medical staff for providing the accurate treatment to patient. After the classification of tumor, the location of tumor is identified by the system which is effectively help medical staff for providing the proper treatment to the patient, or there is a need of tumor surgery then it will provide huge help to doctors.

\section{Results and Discussion}

The proposed technique was implemented using Python programming language. The Convolutional Neural Network (CNN) algorithm was implemented for the classification of various images. We have taken 1000 MRI images from Kaggle dataset and we have used $75 \%$ of input images for training and $30 \%$ for testing. The performance of the proposed technique was evaluated using different measurement parameters. The Accuracy was calculated using following equations.

$$
\begin{gathered}
\text { Presicion }=\frac{\mathrm{TP}}{\mathrm{TP}+\mathrm{FP}} \\
\text { Recall }=\frac{\mathrm{TP}}{\mathrm{TP}+\mathrm{FN}} \\
\text { Specificity }=\frac{\mathrm{TN}}{\mathrm{TN}+\mathrm{FP}}
\end{gathered}
$$




$$
\text { Accuracy }=\frac{\mathrm{TP}+\mathrm{TN}}{\mathrm{TP}+\mathrm{FP}+\mathrm{FN}+\mathrm{TN}}
$$

TP-True Positive, TN-True Negative, FP-False Positive and FN-False Negative
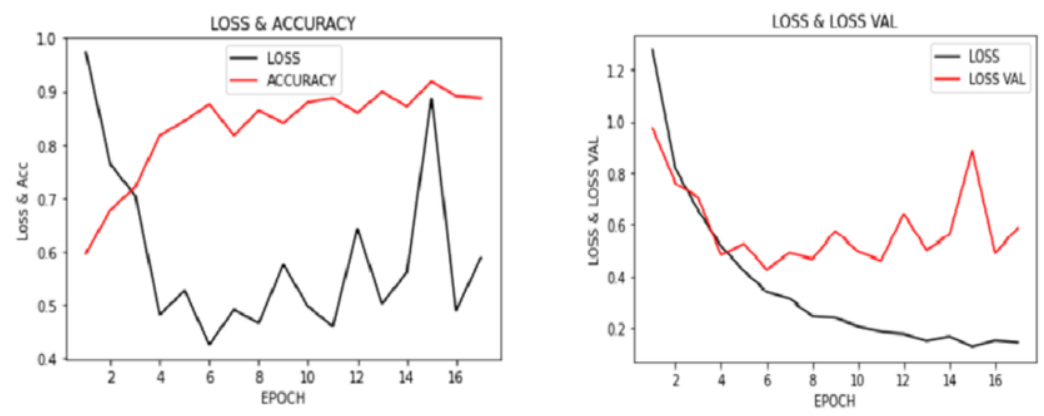

Figure 2. Loss and Accuracy value of the training model for the data set

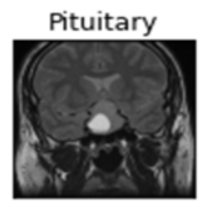

Meningioma

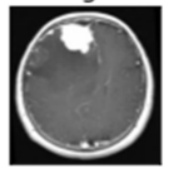

Meningioma
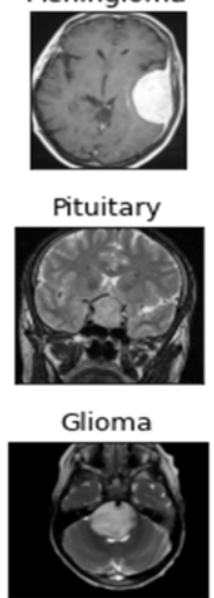

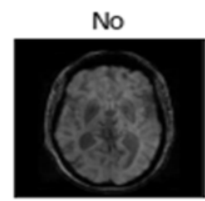

No

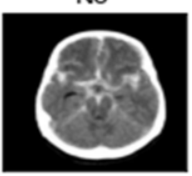

Meningioma
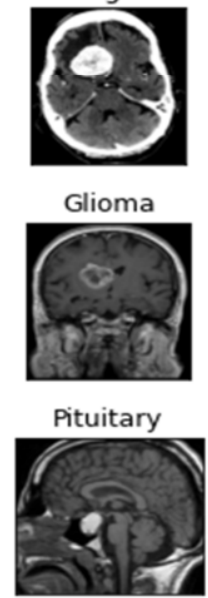

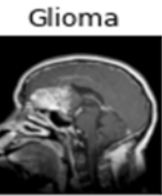

Glioma

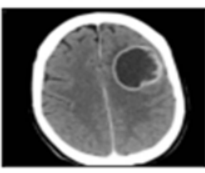

No

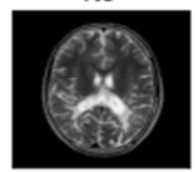

Meningioma

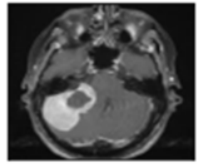

Meningioma

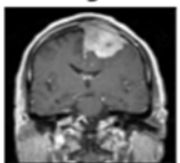

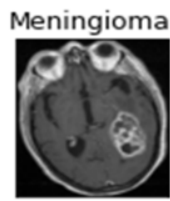

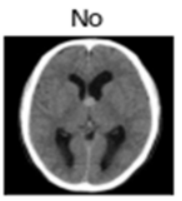

No
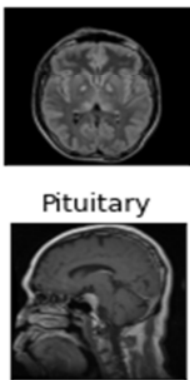

No

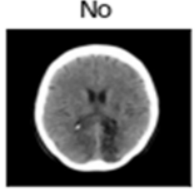

Meningioma

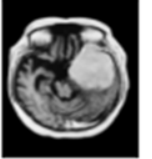

No

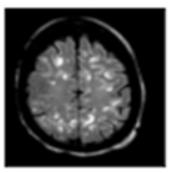

Pituitary

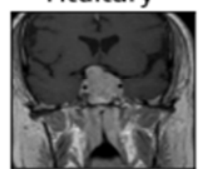

Meningioma

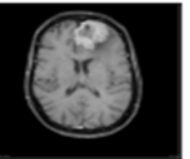

Meningioma

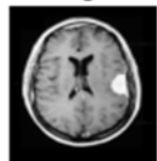

Figure 3. Classification of input data based on the classes i.e. Glioma, Pituitary, Meningioma and Normal. 


\section{Conclusion}

As we all know the brain tumor is one of the dangerous diseases. It can cause the human death. This disease is not possible to detect early using manual processing. So, in this paper we used some Deep Learning models to detect tumor as early as possible. In this paper we used $\mathrm{CNN}$ algorithm which is very important for image processing and classification. In CNN there are three main layers i.e., convolutional layer, activation layer and pooling layer. These all layers are interconnected so that CNN can process and perceive data in order to classify images. Based on classification prediction is done. Another module is used i.e., localization using specific object detection. Defected area of brain will be highlighted using localization.

\section{References}

[1]. Ming Li, LishanKuang, Shuhuaxu and ZhanguoSha, "Brain Tumor Detection Based on Multimodal Fusion and Convolutional Neural Network," IEEE Access,dec 9, 2019.

[2]. S. Khajanchi and S. Banerjee, "A strategy of optimal efficacy of T11target structure in the treatment of brain Tumor,” J. Biol. Syst., vol. 27, no. 1, pp. 1-31, 2019.

[3]. M. A. Khan, I. U. Lali, and A. Rehman, "Brain Tumor detection and classification: A framework of marker-based watershed algorithm andmultilevel priority features selection,’’ Microsc. Res. Technique, vol. 82, no. 6, pp. 909-922, 2019.

[4]. T. Cao, W. Wang, S. Tighe, and S. Wang, "Crack image detection based on fractional differential and fractal dimension,'” IET Comput. Vis., vol. 13, no. 1, pp. 79-85, Feb. 2019.

[5]. M. Ramalho, A. P. Matos, and M. Alobaidy, "Magnetic resonance imaging of the cirrhotic liver: Diagnosis of hepatocellular carcinoma and evaluation of response to treatment-Part 1," RadiologiaBrasileira, vol. 50,no. 1, pp. 38-47, 2017.

[6]. Q. Li, Z. Gao, and Q. Wang, "Glioma segmentation with a uni-fied algorithm in multimodal MRI images,' IEEE Access, vol. 6, pp. 9543- 9553, 2018.

[7]. W. Wu, A. Y. C. Chen, and L. Zhao, "Brain tumor detection and segmentation in a CRF framework with pixel-pairwise affinity and superpixel-level features,' Int. J. Comput. Assist. Radiol.Surg., vol. 9, no. 2, pp. 241-253, 2014.

[8]. Potharaju, S. P., Sreedevi, M., \& Amiripalli, S. S. (2019). An Ensemble Feature Selection Framework of Sonar Targets Using Symmetrical Uncertainty and Multi-Layer Perceptron (SU-MLP). In Cognitive Informatics and Soft Computing (pp. 247-256). Springer, Singapore

[9]. Y.S. Deshmukh, P. Kumar, R. Karan and S.K. Singh, "Breast Cancer Detection based Feature Optimization using Firefly Algorithm and Ensemble Classifier", 2021 International Conference on Artificial Intelligence and smart system(ICAIS) IEEE, pp. 1048-1054.

[10]. Kumar, A., Vengatesan, K., Vincent, R., Rajesh, M., \& Singhal, A. (2019). A novel Arp approach for cloud resource management. International Journal of Recent Technology and Engineering (IJRTE), 7(6).

[11]. Potharaju, S. P., \& Sreedevi, M. (2018). A novel cluster of quarter feature selection based on symmetrical uncertainty. Gazi University Journal of Science, 31(2), 456-470. 